\title{
Corrigendum: X-ray holography with a customizable reference
}

Andrew V. Martin, Adrian J. D'Alfonso, Fenglin Wang, Richard Bean, Flavio Capotondi, Richard A. Kirian, Emanuele Pedersoli, Lorenzo Raimondi, Francesco Stellato, Chun Hong Yoon \& Henry N. Chapman

Nature Communications 5:4661 doi: 10.1038/ncomms5661 (2014); Published 22 Aug 2014; Updated 16 Oct 2014

The original version of this Article contained an error in the spelling of the author Emanuele Pedersoli, which was incorrectly given as Emmanuele Pedersoli. This has now been corrected in both the PDF and HTML versions of the Article. 